Check for updates

Cite this: Phys. Chem. Chem. Phys., $2018,20,29989$

Received 8th October 2018 Accepted 12th November 2018 DOI: $10.1039 / c 8 c p 06264 a$ rsc.li/pccp

\section{Kinetic arrest of front transformation to gain access to the bulk glass transition in ultrathin films of vapour-deposited glasses $\dagger$}

\author{
Joan Ràfols-Ribé, (D) Ana Vila-Costa, Cristian Rodríguez-Tinoco, \\ Aitor F. Lopeandía, (D) Javier Rodríguez-Viejo and Marta Gonzalez-Silveira (D)*
}

\begin{abstract}
Physical vapour deposition has emerged as the technique to obtain glasses of unbeatable stability. However, vapour deposited glasses exhibit a different transformation mechanism to ordinary glasses produced from liquid. Vapour deposited glasses of different thermodynamic stability, from ultrastable to those similar to ordinary glasses, transform into the liquid state via front propagation starting at the most mobile surfaces/ interfaces, at least for the first stages of the transformation, eventually dynamiting the high thermal stability achieved for some of these glasses. A previous study showed that it was possible to avoid this transformation front by capping the films with a higher $T_{\mathrm{g}}$ material. We show here fast calorimetry measurements on TPD and IMC vapour deposited glasses capped respectively with TCTA and TPD. This capped configuration is very effective in suppressing the heterogeneous transformation of the stable glasses into the supercooled liquid and shifts the devitrification temperature to much higher values, where the bulk homogeneous mechanism becomes active. This approach may be useful to further study the bulk glass transition in thin films.
\end{abstract}

\section{Introduction}

It has been thoroughly demonstrated how highly stable glasses can be effectively produced by vapour depositing them at the appropriate substrate temperature and slow deposition rates. ${ }^{1}$ Although the thermodynamic stability of an ultrastable glass cannot be further enhanced within experimental time scales, as it already has the properties of an equivalent hyper-aged glass, ${ }^{2}$ the kinetic stability of these glasses is dominated by the surface-initiated growth front mechanism they exhibit. ${ }^{3}$ For instance, a $20 \mathrm{~nm}$ thick layer of an ultrastable glass will take one-fifth less time to fully transform into supercooled liquid than a $100 \mathrm{~nm}$ thick layer at the same annealing temperature. ${ }^{4}$ Thus, the heterogeneous transformation can reduce the high kinetic stability achieved for these glasses. This represents one of the major limitations for the conceivable applications of the ultrastable vapour deposited glasses. Both, surface crystallization ${ }^{5-7}$ and the amount of time at a given temperature these glasses can endure before transforming to the supercooled liquid, are what will, in the end, determine the practical applications of these glasses. ${ }^{8}$

The transformation into the supercooled liquid of stable glasses is expected to begin at surfaces where the mobility is high. It can be free surfaces, ${ }^{9-13}$ but also interfaces with a more

Group of Nanomaterials and Microsystems, Physics Department,

Universitat Autònoma de Barcelona, Barcelona, 08193 - Bellaterra, Spain.

E-mail: marta.gonzalez@uab.cat

$\dagger$ Electronic supplementary information (ESI) available. See DOI: 10.1039/c8cp06264a mobile material, for instance, a layer of conventional glass. ${ }^{14}$ Also, a slower propagating front starting at the interface with the substrate has been identified for IMC and TPD using spectroscopic ellipsometry during an isothermal annealing protocol. ${ }^{15,16}$ The transformation of stable glasses into the supercooled liquid can be understood in terms of the 'kinetic facilitation' concept. That is, the idea that an immobile region can become mobile only if mobility is present in an adjacent region. ${ }^{17}$ Facilitated kinetic Ising models have already been successfully used to reproduce the behaviour of ultrastable glasses, more specifically, in predicting the constant velocity growth front. ${ }^{11,13}$ Hence, adopting the facilitated kinetics point of view, we can think of further manipulating the properties of the previously studied glasses. The basic idea is that if the highly-mobile surface layers are blocked-somehow-then the growth front transformation mechanism is suppressed. This methodology was already applied by Sepúlveda et al. ${ }^{18}$ who capped the free surface of a stable glass of indomethacin with a stable glass with higher glass transition temperature $\left(T_{\mathrm{g}}\right)$ and were able to avoid, in this way, the formation of a growth front. By eliminating-apparently - the surface mobility, they increased the samples' kinetic stability by further delaying the transformation into the supercooled liquid.

An application field especially interested in increasing the thermal stability of vapour deposited amorphous thin films is the OLED industry. ${ }^{8}$ There, from two to several thin organic layers in the range of tenths of nanometres thick are commonly 
used for building the optoelectronic devices, all of them being generally in their glassy state. The glassy layers in OLED devices do not have any free surfaces, and therefore, their thermal stability will be determined by the layer with the lowest $T_{\mathrm{g}}$ material, among other factors. ${ }^{19,20}$ Thus, it is of vital importance for this field to understand the effect of capping on the thermal stability of organic semiconductor glassy layers.

In this work we use quasi adiabatic fast-scanning membranebased nanocalorimetry ${ }^{21}$ to study different films and capping configurations. The high sensitivity of the technique together with the use of fast heating rates, allows the study of the devitrification process of vapour deposited thin film glasses in a temperature range not accessible by other techniques. ${ }^{22}$ With the proper data treatment, nanocalorimetry gives access not only to the thermodynamic and kinetic stability of the glass but also to the transformation mechanism ${ }^{22}$ or the relaxation time as a function of temperature. ${ }^{23}$

To carry out this study, we have chosen the organic semiconductor TPD ( $N, N^{\prime}$-bis(3-methylphenyl)- $N, N^{\prime}$-diphenylbenzidine), and the pharmaceutical molecule IMC (1-(4-Chlorobenzoyl)-5methoxy-2-methyl-3-indoleacetic acid), as they form glasses with enhanced stability and have been widely characterised as a function of deposition temperature..$^{2,3,15,16,22,24-30}$ IMC shows a maximum of stability when deposited around $266 \mathrm{~K}\left(0.85 T_{\mathrm{g}}\right)^{26}$ and a transition into the supercooled liquid governed in the first stages by a transformation front whose velocity depends on the deposition conditions of the IMC glass. ${ }^{15,26}$ Spectroscopic ellipsometry measurements of thin film TPD glasses have shown that TPD also transforms via a propagating front under isothermal conditions. ${ }^{16}$ To eliminate the surface mobility, we must use a material with lower mobility, i.e. a material with a higher $T_{\mathrm{g}}$. The chosen materials are the organic semiconductor TCTA (tris(4-carbazoyl-9-ylphenyl)amine), with its $T_{\mathrm{g}}$ at $424 \mathrm{~K}$, i.e. $91 \mathrm{~K}$ above the glass transition temperature of TPD $\left(T_{\mathrm{g}}=\right.$ $333 \mathrm{~K}$ ), and TPD itself for capping the IMC films, with a $T_{\mathrm{g}} 19 \mathrm{~K}$ above the glass transition of IMC. Both TPD and TCTA glass transition temperatures have been obtained from standard calorimetry measurements by cooling and heating the materials at $10 \mathrm{~K} \mathrm{~min}^{-1}$. In this study we present the effect of positioning the capping layers at the top or bottom, or on both sides of the glassy films under evaluation. In this way, we can explore the impact of this extra low-mobility layer on the transformation mechanism of the TPD and IMC glasses. Moreover, we vary the deposition temperature of the TPD and IMC sandwiched glasses to study the transformation mechanism in glasses of different stability. The thermal stability of TPD and TCTA as a function of the deposition temperature has also been studied and can be found in the ESI. $\dagger$ The importance of this step relies on the fact that, when evaporated as capping layers, the stability of the capping film will condition its effectiveness at blocking the adjacent layer.

\section{Experimental method}

Samples are prepared and measured in situ in a UHV chamber with a base pressure of $3 \times 10^{-8}$ mbar. A liquid nitrogen cold trap is installed to quench water molecules, improving the vacuum quality. The chamber is equipped with two effusion cells which allow creating multilayers of two organic materials without breaking the vacuum. Samples are deposited directly onto the calorimeter membrane which is fed with a constant intensity to keep the sensing area at a given temperature. Deposition temperatures vary depending on the experiment. In the case of the TPD/TCTA multilayer system, TPD has been deposited at 3 different temperatures, namely $250 \mathrm{~K}, 285 \mathrm{~K}$ and $325 \mathrm{~K}$, while TCTA has been deposited always at $285 \mathrm{~K}$. This temperature yields a relatively stable glass for TCTA (see ESI $\dagger$ ). Although this temperature is $139 \mathrm{~K}$ below TCTA's $T_{\mathrm{g}}$, some ageing takes place in the bottom TCTA film during the deposition of TPD at $325 \mathrm{~K}$ (see ESI $\dagger$ ). The small aging observed does not, apparently, modify the capping properties of this film. A different approach has been used for the IMC/TPD multilayer system. Both IMC and TPD have been deposited at the same temperature, namely $266 \mathrm{~K}, 300 \mathrm{~K}$ and $310 \mathrm{~K}$ depending on the stability under study.

The thickness of the films is controlled by a quartz crystal microbalance (QCM) previously calibrated and located nearby the sample holder. For the TPD/TCTA system, the growth rates have been $0.08 \pm 0.01 \mathrm{~nm} \mathrm{~s}^{-1}$ for TPD films and $0.07 \pm 0.01 \mathrm{~nm} \mathrm{~s}^{-1}$ for TCTA. For the IMC/TPD films, the rates have been of $0.06 \pm$ $0.01 \mathrm{~nm} \mathrm{~s}^{-1}$ for both materials. IMC crystalline powder $(99.9 \%$ purity) was acquired from Sigma-Aldrich. TPD was purchased from Cymit Química S. L. with a purity level above 99\%. TCTA was obtained from their crystalline powder phase (sublimated grade $>99.0 \%$ ) from TCI Chemicals. All materials were used as received without further purification.

The heat capacity of the single and multilayered films was measured by quasi-adiabatic fast scanning nanocalorimetry in differential mode. ${ }^{21,31}$ This technique relies on the use of membrane-based micro-calorimeters to attain high heating rates $\left(3 \times 10^{4} \mathrm{~K} \mathrm{~s}^{-1}\right)$, achieving very high sensitivities. ${ }^{32}$ Samples are deposited on an aluminium plate of $200 \mathrm{~nm}$ previously grown on the sensing area of the device $\left(1 \mathrm{~mm}^{2}\right)$. The aluminium layer homogenizes the temperature of the sensing area improving the temperature resolution of the calorimetric signal.

\section{Results and discussion}

\section{Capping configurations}

The first step is to study the impact of capping a thin layer of a low- $T_{\mathrm{g}}$ material (TPD or IMC) with a high- $T_{\mathrm{g}}$ material (TCTA or TPD, respectively), in different configurations, as shown in Fig. 1. First and for comparison purposes, we prepare single layers of TPD and IMC (a). The second step is to evaluate the effect of having one single high- $T_{\mathrm{g}}$ material layer either between substrate and the film under study or capping the free surface exposed to the vacuum (b and $c$ ). The final configuration corresponds to the studied glass capped at both sides (d).

Fig. 2 shows the heat capacity scans corresponding to the four multilayer configurations for TPD/TCTA and for IMC/TPD when the low- $T_{\mathrm{g}}$ material is deposited in its ultrastable form, 


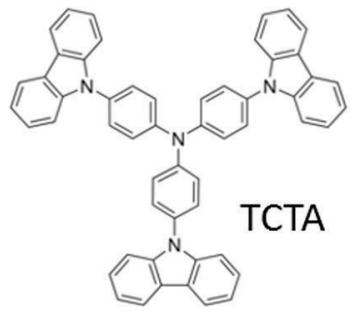

(a)

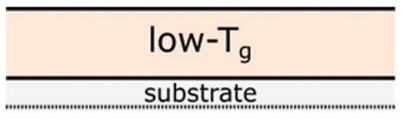

(c)

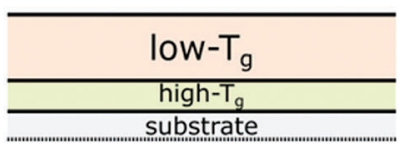

\begin{tabular}{|c|}
\hline high- $T_{q}$ \\
\hline low-Tg \\
\hline$\frac{\text { high-T }}{\text { substrate }}$ \\
\hline
\end{tabular}

Fig. 1 Molecular structure of TCTA, IMC and TPD. (a-d) Capping configurations.

i.e. at $0.85 T_{\mathrm{g}}$. We see two distinct scenarios: (i) if a high- $T_{\mathrm{g}}$ capping layer covers the free surface of the low- $T_{\mathrm{g}}$ material layer (Fig. 2, up and down triangles), the onset of devitrification shifts to much higher temperatures-about $35 \mathrm{~K}$ for TPD and $22 \mathrm{~K}$ for IMC. (ii) If the high- $T_{\mathrm{g}}$ material is only placed at the interface between the substrate and the film, it results in an equivalent calorimetric trace as having a single layer of the low$T_{\mathrm{g}}$ material deposited directly on the nanocalorimeter sensor (Fig. 2, filled squares and circles respectively). The small differences are due to a different thickness of the films or the overlap with the high- $T_{\mathrm{g}}$ glass transition peak. Considering that, when deposited as a single film, this transition has been identified as a front mediated transformation (see C. RodríguezTinoco et $a l^{26}$ for IMC and $\mathrm{ESI}^{\dagger} \dagger$ for TPD), this is a clear indication that our TPD and IMC ultrastable glass films devitrify via one single free surface-initiated front.

From Fig. 2 we can see that the capping has also some effect on the capping material, especially visible in the case of the TPD/TCTA system. In the single TCTA scan, we see the onset of the devitrification peak around $460 \mathrm{~K}$ for a $17 \mathrm{~nm}$ thick layer (Fig. 2a, open squares). However, when TCTA is deposited in contact with a TPD layer, we do not see the calorimetric trace corresponding to its devitrification (Fig. 2a, triangles and circles). A better insight to this phenomenon can be found in Fig. 3, where we plot the heat capacity of the TCTA/TPD/TCTA configuration for TPD samples deposited at $0.85 T_{\mathrm{g}}$ and at $T_{\mathrm{g}}$ together with the previous single scan of TCTA. The calorimetric trace of the TPD sample deposited at $T_{\mathrm{g}}$ exhibits two well-defined transitions. The first one is the expected transition for TPD deposited at $T_{\mathrm{g}}$, the second one corresponds to the devitrification
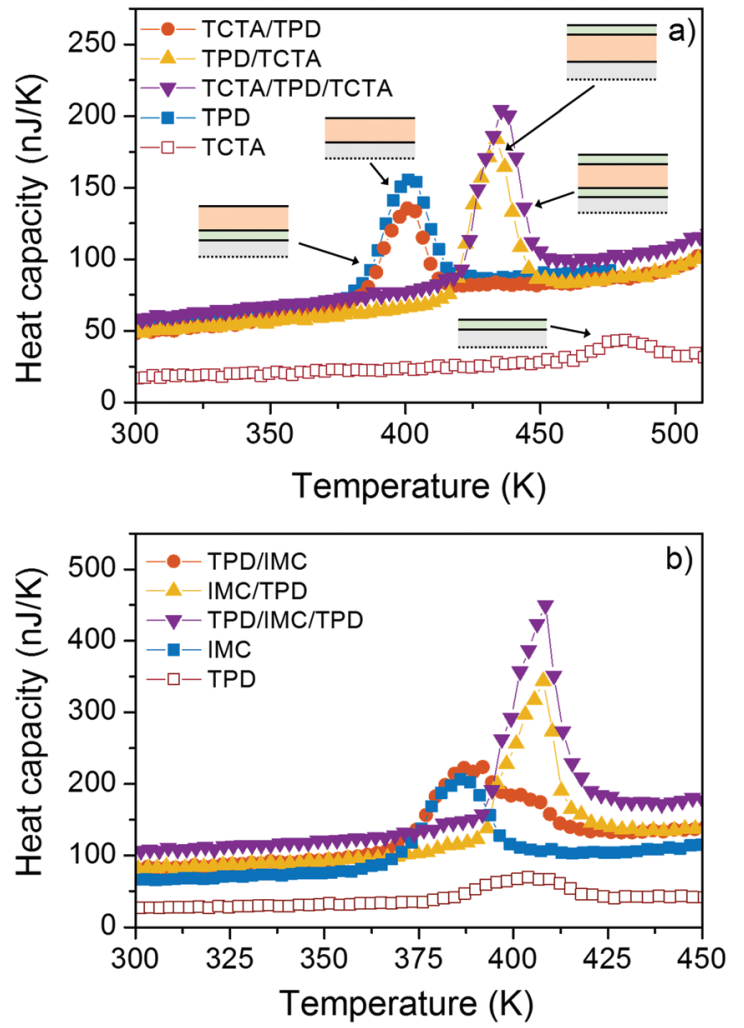

Fig. 2 Heat capacity scans of the ultrastable glass of TPD ( $T_{\text {dep }}=285 \mathrm{~K}$; upper panel) and IMC ( $T_{\text {dep }}=266 \mathrm{~K}$; lower panel) for the different configurations presented in Fig. 1 as indicated in the legend. (a) For the TPD/TCTA samples, the thickness of the TCTA layers is of $17 \mathrm{~nm}, 44 \mathrm{~nm}$ for the single TPD and $34 \mathrm{~nm}$ for the TPD in any of the TPD/TCTA multilayer configurations. (b) For the IMC/TPD samples, IMC is $40 \mathrm{~nm}$ thick and TPD $20 \mathrm{~nm}$ thick in all cases.

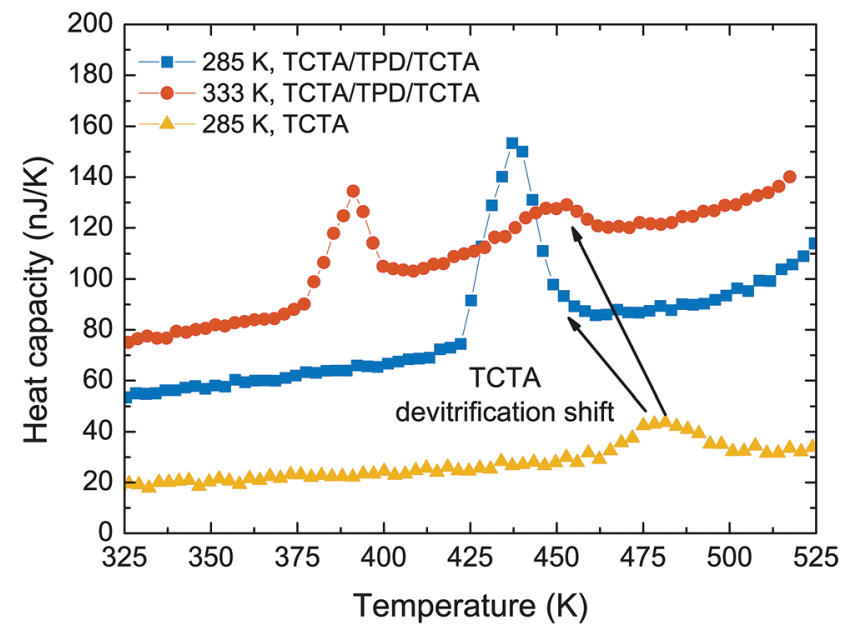

Fig. 3 Heat capacity for capped TPD layers with the sandwich configuration deposited at $T_{\mathrm{g}}$ (circles) and $0.85 T_{\mathrm{g}}$ (squares) and for a single TCTA layer deposited at $285 \mathrm{~K}$. The films' thicknesses are in all cases between 18 and $25 \mathrm{~nm}$.

of the TCTA occurring at much lower temperatures than the expected for a single TCTA layer, around $30 \mathrm{~K}$ below. Therefore, it seems likely that in the case of the capped ultrastable glass of TPD, both devitrifications-TPD and TCTA-are overlapped. 
That can also explain the slight differences in the onset and the area seen between the TCTA/TPD/TCTA configuration and the single top-capping layer, because of the double mass of TCTA transforming with respect to the TPD layer overlapped with the glass transition of TPD. In fact, when looking at the curves from configuration TCTA/TPD (Fig. 2, circles), we can infer a small peak just after the devitrification of the TPD sample, around $430 \mathrm{~K}$, which would correspond to the transformation of the TCTA layer. Something similar happens when capping IMC with TPD. Although in this case both the glass transition of single IMC and TPD are already overlapped (see Fig. 2b), the transition of TPD is slightly shifted to lower temperatures when deposited as a capping layer.

The differences between the TCTA devitrification temperatures when arranged as a single layer or following a multilayer configuration with TPD could initially be explained from the kinetic facilitation point of view. ${ }^{17}$ A TCTA vapour deposited layer will start transforming into supercooled liquid in the regions where the mobility is higher, that is, on the free surface of the TCTA single layer. However, when the TCTA surface is in contact with a TPD film, the moment TPD devitrifies, the interface between TCTA and TPD becomes more mobile than TCTA's free surface at that temperature, inducing the transformation of the TCTA layer.

From a slightly different approach, the substantial decrease in the onset temperature of TCTA can also be rationalised in terms of interlayer diffusion. McEwan et $a l^{33}$ showed the mixing process of different glassy layers of organic semiconductor materials using neutron reflectometry measurements. They heated stacks of three organic layers with distinct glass transition temperatures and followed the diffusion profiles. They found out that a diffusion process started when the lowest $T_{\mathrm{g}}$ material was in the supercooled liquid state. The diffusion took place essentially from the high- $T_{\mathrm{g}}$ layer towards the adjacent supercooled liquid. ${ }^{33}$ The resulting intermixed layer exhibited a new $T_{\mathrm{g}}$ in between the original ones. Although their experiments were carried out under isothermal conditions, a similar rationalisation can be made for fastscanning nanocalorimetry. For the TPD/TCTA system, which is more affected by this difference in $T_{\mathrm{g}}$, we can extrapolate and consider that once our capped TPD sample has transformed into the supercooled liquid, TCTA molecules probably start to diffuse into the TPD layer. This process ends with the TCTA layer intermixed with the newly formed layer, still glassy, with a depressed $T_{\mathrm{g}}$ with respect to pure TCTA. Despite the diffusion process in a heating ramp might be slightly more complicated, this simple physical picture can explain the depressed $T_{\mathrm{g}}$ we observe for a TCTA layer in contact with a lower $T_{\mathrm{g}}$ material.

\section{Transformation mechanisms in capped glasses}

Although capping either surface and interface with the substrate or only the surface (configurations d and b respectively, Fig. 1) do not generate major differences in their devitrification process, to reduce the number of factors controlling the devitrification, we will continue our study with the full sandwich configuration. In this way, the sandwiched layers will have two identical interfaces with the substrate and the top layer.
Fig. 2 shows how the devitrification of TPD and IMC layers is delayed when the surface is capped, however, the transformation mechanism under this configuration remains to be confirmed. If a homogeneous mechanism is governing the transformation into the supercooled liquid, we should see, for same stability samples but different thicknesses, how the onset-and the entire peak-of the specific heat curves collapses. ${ }^{4}$ For that purpose, films of different thicknesses of the low- $T_{\mathrm{g}}$ material have been evaporated both as single and sandwiched layer. To evaluate if the transformation mechanism changes depending on the stability of the glass, the same strategy has been used for films obtained at different deposition temperatures. The contribution of the heat capacity of the high- $T_{\mathrm{g}}$ glass is subtracted from each curve to simplify the analysis of the curves corresponding to different sample sizes. However, we must consider the following aspects: (i) the mass from the capping layer must be inferred from the QCM reading since it is not possible to deconvolute the heat capacity signal from the capping and the capped layer separately. (ii) The contribution of the capping layer cannot be subtracted in the whole temperature range of the calorimetric scan. This is because its devitrification occurs at different temperatures depending if it is in its single or multilayered configuration. Therefore, in the region after the transition, the capping layer contribution cannot be properly subtracted. (iii) Consequently, the normalisation we are performing accounts neither for the area of the peak nor the specific heat in the liquid region. (iv) Once the subtraction is performed, the mass of the capped layer can be then inferred from the heat capacity in the glass region of the resulting curve, instead of using the supercooled liquid region as was used in previous measurements. ${ }^{4,22,26}$ This procedure yields thicknesses in agreement with the values measured by the QCM during the evaporation.

In Fig. 4 we can see the specific heat trace for three different thicknesses of a TPD glass deposited at $285 \mathrm{~K}, 250 \mathrm{~K}$ and $325 \mathrm{~K}$ for single and capped layers. While the single layers show the onset shift typical in heterogeneous transformations ${ }^{4}$ (void symbols), the onset and peak of the capped glass collapse regardless of the sample's thickness, indicative of a homogeneous transformation. The small variations in the area of the peak are an artefact produced by the different TPD/TCTA mass ratios that each sample has and which the normalisation is not taking into account. Equivalent results are obtained for IMC deposited at three more temperatures. The corresponding specific heat curves are shown in the ESI $\dagger$ for deposition temperatures of $266 \mathrm{~K}, 300 \mathrm{~K}$ and $310 \mathrm{~K}$. These results corroborate that the transformation of thin film glasses which surface has been kinetically arrested is taking place via a homogeneous mechanism, not only in the case of the ultrastable glass but also for less stable glasses.

From the kinetic facilitation point of view, we are, in fact, preventing the start of the propagation front by arresting the higher mobility region. Previous work from Sepúlveda et al. showed that the propagating transformation front of ultrastable IMC glasses could be impeded with a capping layer of a higher $T_{\mathrm{g}}$ material, TNB in their case. ${ }^{18}$ There, they used deuterated IMC layers to follow the concentration profile during 


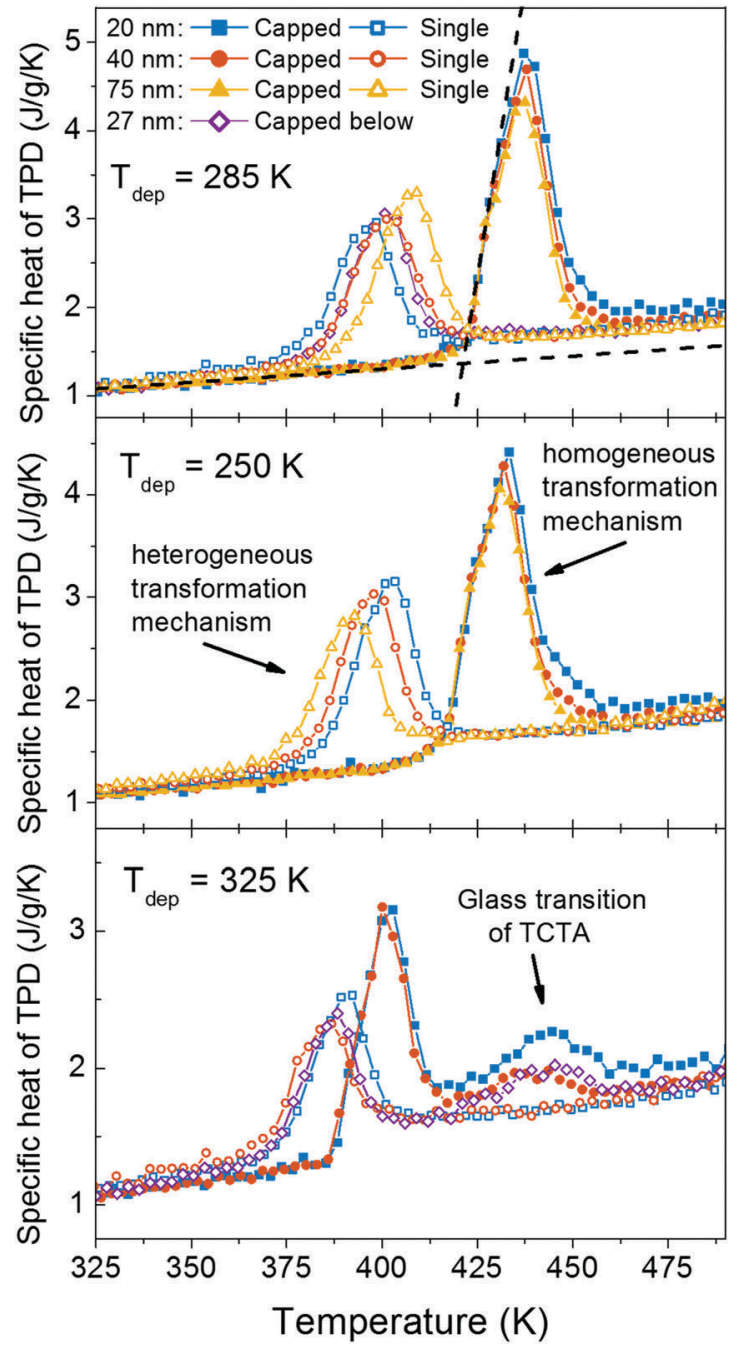

Fig. 4 Calorimetric trace glasses of TPD deposited at (a) $285 \mathrm{~K}\left(0.85 \mathrm{~T}_{\mathrm{g}}\right)$, (b) $250 \mathrm{~K}\left(0.75 \mathrm{~T}_{\mathrm{g}}\right)$ and $(\mathrm{c}) 325 \mathrm{~K}\left(0.98 \mathrm{~T}_{\mathrm{g}}\right)$. The curves correspond to single (void symbols) and capped layers with configuration TCTA/TPD/TCTA (filled symbols). Different symbols indicate different TPD thicknesses, as labelled in the legend. Dispersion in thickness is maximum $15 \%$ from the values in the legend. TCTA layers are between 15 and $17 \mathrm{~nm}$ thick. The diamonds correspond to a TPD/TCTA bilayer configuration. The dashed black lines in panel (a) show how the onset of devitrification for the capped layers is obtained.

an annealing at $T_{\mathrm{g}}+11 \mathrm{~K}$. Although their capped samples eventually transformed into the supercooled liquid, this was accomplished at much longer times than the samples with a free surface. In our case instead, we use temperature-ramping experiments to prove that the growth front can be blocked and the devitrification process delayed. For both TPD and IMC, we see that not only the UG can be capped, but also less stable glasses. In the light of these results with the systems TPD/TCTA and IMC/TPD, together with the ones provided by Sepúlveda et $a l .{ }^{18}$ for the system IMC/TNB, the capping strategy to preclude the front transformation could be generalised to other glass-forming materials. Moreover, it provides us with the opportunity to study the homogeneous transformation of very thin films.

\section{Single front transformation}

Fig. 4 and Fig. S2 (see ESI $\dagger$ ) show that TPD and IMC thin film glasses deposited both above and below $0.85 T_{\mathrm{g}}$, when deposited on top of a glass with lower mobility, behave equivalently to a single layer of the same thickness and stability. These results are entirely equivalent to the ones anticipated for the ultrastable glass. Therefore, we can infer that only a surface-initiated front is responsible for the heterogeneous transformation mechanism of the non-capped TPD and IMC samples irrespectively of their deposition conditions.

The single surface-initiated front we have inferred here for the transformation of stable glasses of TPD and IMC contrasts with the results obtained by Walters et al. ${ }^{16}$ and Dalal et al. ${ }^{15}$ There, they use spectroscopic ellipsometry and a multilayer optical model to trace the evolution of the isothermal transformation into the supercooled liquid of TPD and IMC stable glasses. Their data is best fitted using a two-front model, a surface and a substrate-induced front. However, our data is obtained in a much higher temperature range, about $T_{\mathrm{g}}+40 \mathrm{~K}$ and above, compared to their isothermal measurements at $T_{\mathrm{g}}+$ $15 \mathrm{~K}$ at most. Their substrate-induced front exhibits slower velocities than the surface front. A possible explanation might be that, at our high-temperature range, the surface-initiated front completely dominates the transformation or that our aluminium substrate has a stronger interaction with the organic layer than silicon oxide, the substrate used in the above mentioned studies. In this case, the aluminium would act similarly to what we see for a higher $T_{\mathrm{g}}$ material, blocking this second front. Using SIMS, Sepúlveda et al. ${ }^{14,18}$ did not find either evidence of a secondary front starting at the substrate on IMC and TNB glasses, although they sometimes see a front starting at the (deuterated)IMC/IMC interface. ${ }^{18}$ Adachi et $a l .{ }^{34}$ also used ellipsometry to investigate the transformation mechanism of organic semiconductors molecules. Their best fit of the experimental data is provided by a combined model of a surface-initiated front and a homogeneous transformation. Although this latter combined model was not tested by Walters et $a l .{ }^{16}$ for TPD, it might also provide a plausible explanation to their double-front best fit.

\section{Kinetic stability of a capped glass}

By capping the TPD layers, we are delaying the onset of devitrification and thus, enhancing the kinetic stability of these glasses. Fig. 5 shows the specific heat trace for samples deposited at seven different deposition temperatures, $T_{\mathrm{d}}$, for single layers of TPD (lower panel) and capped layers with the TCTA/TPD/TCTA geometry (upper panel). A complementary picture can be found in Fig. 6, where we represent the onset of capped and non-capped films (of similar thickness) as a function of deposition temperature. In the case of the single layers, the heterogeneous mechanism is the limiting factor for increasing the kinetic stability. For instance, the difference in the onset of devitrification for single layers between the sample deposited at $T_{\mathrm{g}}$ and the UG is around $13 \mathrm{~K}$, for $c a .40 \mathrm{~nm}$ thick layers. Of course, this is an apparent onset due to the heterogeneous transformation mechanism and the specific heat 


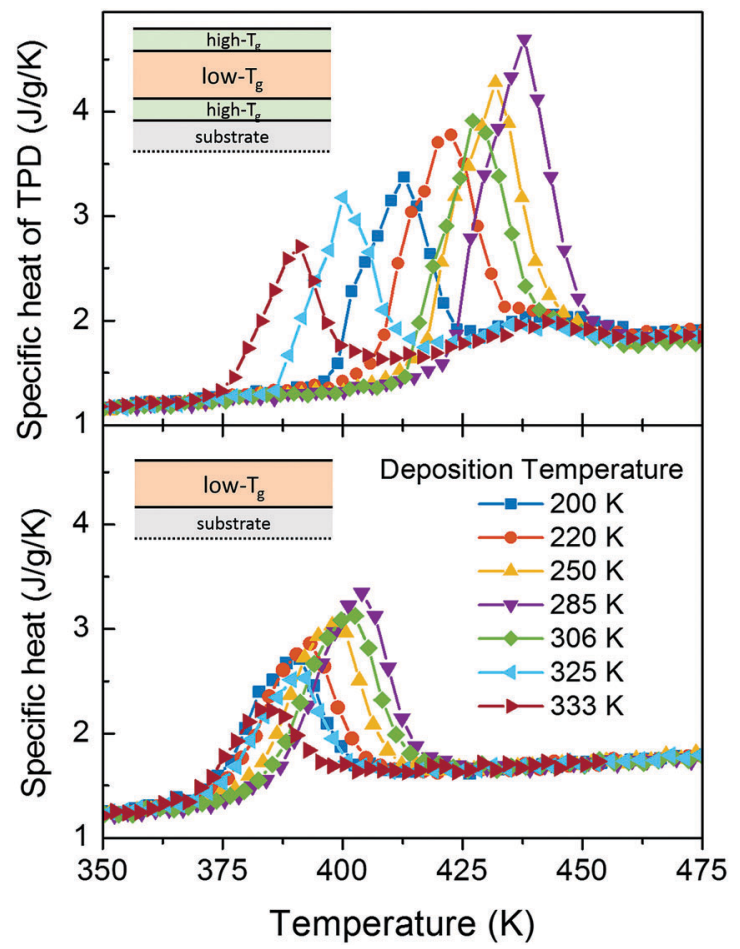

Fig. 5 Calorimetric trace for capped (upper panel) and single (lower panel) TPD samples deposited at different temperatures. The calorimetric trace of the top panel is normalised to the TPD mass, before the subtraction of the TCTA glass contribution to the heat capacity. The deposition temperature is indicated at the lower legend (colour and symbol scheme valid for both graphs). The TPD thicknesses in the TCTA/TPD/TCTA geometry are $45 \mathrm{~nm}$ with a total dispersion between samples of $\pm 13 \%$ (upper panel). The thickness of the single TPD layers is $42 \mathrm{~nm}$ with a total dispersion of $\pm 16 \%$.

representation of the data. This difference can become larger when comparing samples with different thicknesses. However, when capped, the onset difference between the same two samples is expanded up to $44 \mathrm{~K}$. On the contrary, the onset of devitrification of the sample deposited at $T_{\mathrm{g}}$ is practically the same both for the single and the capped film-indicating a small crossover length of few nanometres, defining crossover length as the distance that the transformation front has travelled before the homogeneous transition mechanism dominates the transformation. ${ }^{3}$ This small crossover length for low stability glasses is not in agreement with the measurements from Bhattacharya and Sadtchenko, ${ }^{35}$ who see how even ordinary glasses of methylbenzene and 2-propanol can transform via this propagating front up to several micrometres. Regarding the UG glass, the much larger shift is indicative of a much higher crossover length of probably few hundreds of nanometres at least. Glasses with stabilities in between the ultrastable and the conventional glass show intermediate onset shifts, showing again that the cross-over length is a deposition temperature dependent variable. ${ }^{4}$ Still, this is not an intuitive result. Since ultrastable glasses present slower front velocities, in order to get a larger cross-over length it is necessary that the dynamics of the homogeneous transformation of this type of glasses is far

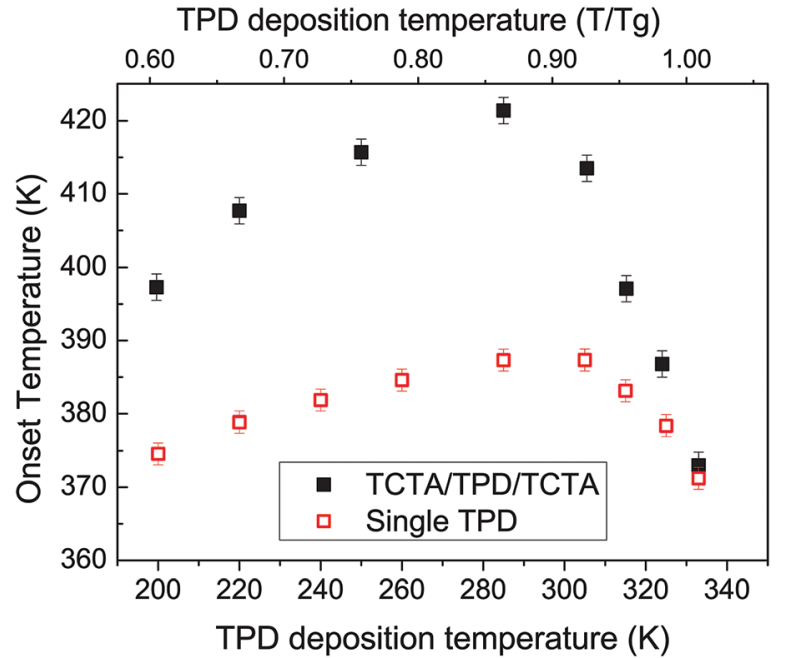

Fig. 6 Onset temperature of the glass transition calorimetric peak for capped (black) and non-capped (red) TPD $42 \mathrm{~nm}$ thick thin film glasses.

slower than that of the less stable glasses. In other words, the difference in front velocity as a function of deposition temperature must be less significant than the change in the dynamics of the bulk. This is clear when looking at the evolution of the onset temperatures for heterogeneous and homogeneous mechanisms (Fig. 6) as a function of deposition temperature.

\section{Conclusions}

We have taken a step forward towards enhancing our understanding of the transformation mechanisms of stable glasses by gaining access to the bulk properties. By capping the stable glasses of TPD and IMC with a higher $T_{\mathrm{g}}$ material we could effectively eliminate the growth front. Using four different multilayer configurations, we have inferred that our single samples of TPD and IMC transform through a single surfaceinitiated front, which contrasts with the substrate-initiated front found by other authors. We have seen how the onset of the devitrification can be delayed up to $40 \mathrm{~K}$ by capping the free surface of an ultrastable glass, while without capping, this delay was limited to $\sim 20 \mathrm{~K}$ and thickness-dependent due to the appearance of a transformation front. These results provide further support to the kinetic facilitation picture in which these highly packed glasses start transforming where the mobility is higher. Once the surface mobility is arrested, the glasses transform via a homogeneous mechanism.

In a typical OLED device, the active organic layers are capped either by the electrodes or by another organic layer, so it is of vital importance to understand the effect of capping on the stability of a glass. We have shown here how the capping strategy delays the transformation of the films, at least in the heating regime. This reinforces the idea of using that stable glasses can be effectively used in designing organic electronic devices with enhanced lifetimes. Further work needs to be done to establish whether the impact of the deposition temperature on the isothermal stability. 


\section{Conflicts of interest}

There are no conflicts to declare.

\section{Acknowledgements}

This work was financially supported by the Spanish Ministry of Economy and Competitiveness and the European Regional Development Fund through projects MAT2013-40896-P and MAT2016-79579-R. Joan Ràfols Ribé was also in receipt of a FPU grant from the Spanish Ministry of Education, Culture and Sport. We thank the support in the microfabrication of the calorimeters by Ll. Abad from IMB-CNM-CSIC and the technical assistance of Theo Bijvoets from Rivac Technology B. V.

\section{References}

1 M. D. Ediger, J. Chem. Phys., 2017, 147, 210901.

2 K. L. Kearns, S. F. Swallen and M. D. Ediger, J. Phys. Chem. B, 2008, 112, 4934-4942.

3 K. L. Kearns, M. D. Ediger, H. Huth and C. Schick, J. Phys. Chem. Lett., 2010, 1, 388-392.

4 J. Ràfols-Ribé, M. Gonzalez-Silveira, C. Rodríguez-Tinoco and J. Rodríguez-Viejo, Phys. Chem. Chem. Phys., 2017, 19, 11089-11097.

5 C. Rodríguez-Tinoco, M. Gonzalez-Silveira, J. Ràfols-Ribé, G. Garcia and J. Rodríguez-Viejo, J. Non-Cryst. Solids, 2015, 407, 256.

6 W. Zhang, C. W. Brian and L. Yu, J. Phys. Chem. B, 2015, 119, 5071-5078.

7 Y. Sun, L. Zhu, K. L. Kearns, M. D. Ediger and L. Yu, Proc. Natl. Acad. Sci. U. S. A., 2011, 108, 5990-5995.

8 J. Ràfols-Ribé, P.-A. Will, C. Hänisch, M. Gonzalez-Silveira, S. Lenk, J. Rodríguez-Viejo and S. Reineke, Sci. Adv., 2018, 4, eaar8332.

9 C. R. Daley, Z. Fakhraai, M. D. Ediger and J. A. Forrest, Soft Matter, 2012, 8, 2206.

10 L. Zhu, C. W. Brian, S. F. Swallen, P. T. Straus, M. D. Ediger and L. Yu, Phys. Rev. Lett., 2011, 106, 256103.

11 S. Léonard and P. Harrowell, J. Chem. Phys., 2010, 133, 244502.

12 G. M. Hocky, L. Berthier and D. R. Reichman, J. Chem. Phys., 2014, 141, 224503.

13 R. Gutiérrez and J. P. Garrahan, J. Stat. Mech.: Theory Exp., 2016, 074005.

14 A. Sepúlveda, S. F. Swallen, L. A. Kopff, R. J. McMahon and M. D. Ediger, J. Chem. Phys., 2012, 137, 204508.
15 S. S. Dalal, Z. Fakhraai and M. D. Ediger, J. Phys. Chem. B, 2013, 117, 15415-15425.

16 D. M. Walters, R. Richert and M. D. Ediger, J. Chem. Phys., 2015, 142, 134504.

17 S. Butler and P. Harrowell, J. Chem. Phys., 1991, 95, 4454.

18 A. Sepúlveda, S. F. Swallen and M. D. Ediger, J. Chem. Phys., 2013, 138, $12 \mathrm{~A} 517$.

19 S. Scholz, D. Kondakov, B. Lüssem and K. Leo, Chem. Rev., 2015, 115, 8449-8503.

20 X. Zhou, J. He, L. S. Liao, M. Lu, X. M. Ding, X. Y. Hou, X. M. Zhang, X. Q. He and S. T. Lee, Adv. Mater., 2000, 12, 265-269.

21 J. Rodríguez-Viejo and A. F. Lopeandía, Fast Scanning Calorimetry, Springer International Publishing, Cham, 2016, pp. 105-149.

22 C. Rodríguez-Tinoco, M. Gonzalez-Silveira, J. Ràfols-Ribé, A. F. Lopeandía, M. T. Clavaguera-Mora and J. RodríguezViejo, J. Phys. Chem. B, 2014, 118, 10795-10801.

23 C. Rodríguez-Tinoco, J. Ràfols-Ribé, M. González-Silveira and J. Rodríguez-Viejo, Sci. Rep., 2016, 6, 35607.

24 A. Gujral, K. A. O'Hara, M. F. Toney, M. L. Chabinyc and M. D. Ediger, Chem. Mater., 2015, 27, 3341-3348.

25 J. Ràfols-Ribé, R. Dettori, P. Ferrando-Villalba, M. GonzalezSilveira, L. Abad, A. F. Lopeandía, L. Colombo and J. Rodríguez-Viejo, Phys. Rev. Mater., 2018, 2, 035603.

26 C. Rodríguez-Tinoco, M. Gonzalez-Silveira, J. Ràfols-Ribé, A. F. Lopeandía and J. Rodríguez-Viejo, Phys. Chem. Chem. Phys., 2015, 17, 31195-31201.

27 K. L. Kearns, K. R. Whitaker, M. D. Ediger, H. Huth and C. Schick, J. Chem. Phys., 2010, 133, 014702.

28 Z. Chen, A. Sepúlveda, M. D. Ediger and R. Richert, J. Chem. Phys., 2013, 138, 12 A519.

29 S. S. Dalal, D. M. Walters, I. Lyubimov, J. J. de Pablo and M. D. Ediger, Proc. Natl. Acad. Sci. U. S. A., 2015, 112, 4227-4232.

30 K. J. Dawson, K. L. Kearns, M. D. Ediger, M. J. Sacchetti and G. D. Zografi, J. Phys. Chem. B, 2009, 113, 2422-2427.

31 M. Y. Efremov, E. A. Olson, M. Zhang, S. L. Lai, F. Schiettekatte, Z. S. Zhang and L. H. Allen, Thermochim. Acta, 2004, 412, 13-23.

32 E. León-Gutierrez, G. Garcia, A. F. Lopeandia, J. Fraxedas, M. T. Clavaguera-Mora and J. Rodríguez-Viejo, J. Chem. Phys., 2008, 129, 181101.

33 J. A. McEwan, A. J. Clulow, A. Nelson, N. R. Yepuri, P. L. Burn and I. R. Gentle, ACS Appl. Mater. Interfaces, 2017, 9, 14153-14161.

34 T. Komino, H. Nomura, M. Yahiro and C. Adachi, J. Phys. Chem. C, 2012, 116, 11584-11588.

35 U. Cubeta, D. Bhattacharya and V. Sadtchenko, J. Chem. Phys., 2017, 147, 071101. 